\title{
CAN THERE BE NECESSARY CONNECTIONS BETWEEN SUCCESSIVE EVENTS?
}

\section{NICHOLAS MAXWELL}

First published in The British Journal for the Philosophy of Science, vol. 19, 1968, pp. 1-25, and reprinted in R. Swinburne, ed., The Justification of Induction, Oxford University Press, Oxford, 1974, pp. 149-174

THE aim of this paper is to refute Hume's contention that there cannot be logically necessary connections between successive events. I intend to establish, in other words, not 'Logically necessary connections do exist between successive events', but instead the rather more modest proposition: 'It may be, it is possible, as far as we can ever know for certain, that logically necessary connections do exist between successive events.'

Towards the end of the paper I shall say something about the implications of rejecting Hume's contention.

It should perhaps be noted at the outset that Hume does not, in a sense, deny the idea of necessary connections between events: but he does deny the possibility of the objective existence of logically necessary connections between events. According to Hume the idea results from habit or custom after the observed frequent conjunction of two events. Thus he declares: 'Upon the whole, necessity is something that exists in the mind, not in objects ... Either we have no idea of necessity, or necessity is nothing but that determination of the thought to pass from causes to effects, and from effects to causes, according to their experienced union. ${ }^{11}$ In other words we may, if we wish, hold that Hume does not reject, but merely gives an analysis of, the notion of 'necessary connection', but in this case we must admit that his analysis excludes the possibility of the existence of any kind of logically necessary connections between events. Looked at in this way, the main task of this paper is to analyse, or interpret, "There exists a logically necessary connection between two successive events $\mathrm{E}_{1}$ and $\mathrm{E}_{2}$ ", in such a way that this statement cannot be refuted on a priori grounds.

It must be admitted of course that it is propositions, and not events, that can be logically related or connected, and hence that there is something ill-formed about the assertion "There exists a logically necessary connection between $\mathrm{E}_{1}$ and $\mathrm{E}_{2}$ ". In view of this I suggest that this statement is to be analysed as, or is to be interpreted to mean:

(1) At the time of occurrence of $E_{1}$ there exists that which can only be completely described by propositions, $\mathrm{P}_{1}$, which logically imply propositions, $\mathrm{P}_{2}$, that state that $\mathrm{E}_{2}$ occurs subsequently.

The essential requirement here is that, at the time of occurrence of $E_{1}$, there exists something, $\mathrm{X}$ say, which is such that any propositions which completely describe $\mathrm{X}$, logically imply propositions which state that $\mathrm{E}_{2}$ occurs subsequently. No one denies that propositions, $\mathrm{P}_{3}$ say, which incorporate a description of what exists at the time of occurrence of $\mathrm{E}_{1}$ can logically imply propositions which state that $\mathrm{E}_{2}$ occurs subsequently. But if $\mathrm{P}_{3}$ only incorporate a description of what exists at the time of occurrence of $\mathrm{E}_{1}$, it is possible that, by whittling away at $\mathrm{P}_{3}$, one can arrive at propositions, $\mathrm{P}_{4}$ say, which $(a)$ give just as complete a description as $\mathrm{P}_{3}$ of what exists at the time of occurrence of $\mathrm{E}_{1}(b)$ do not logically imply propositions which state that $\mathrm{E}_{2}$ occurs subsequently. Thus the fact that propositions which incorporate a description of what exists at the time of occurrence of $E_{I}$ can logically imply propositions which state that $E_{2}$ occurs subsequently, does not suffice at all to establish the existence of a logically necessary connection between $E_{1}$ and $E_{2}$ in the sense of (1) above.

As far as I can see, (1) above successfully captures all that anyone could hope or want to mean by 'There exists a logically necessary connection between $\mathrm{E}_{\mathrm{I}}$ and $\mathrm{E}_{2}$ '. Granted the truth of (1), that which exists at the time of occurrence of $E_{1}$ determines the subsequent occurrence of $E_{2}$ 
with logical necessity, in the sense that at the time of occurrence of $E_{1}$ there is that which exists-X say — which is such that any propositions which completely describe $\mathrm{X}$ logically imply propositions which state that $\mathrm{E}_{2}$ occurs subsequently. The 'logical connection' is of course between the propositions $\mathrm{P}_{1}$ and $\mathrm{P}_{2}$, not between the events $\mathrm{E}_{1}$ and $\mathrm{E}_{2}$ : nevertheless this 'logical connection' arises not merely as a result of the way in which we choose to think of, or describe, $\mathrm{E}_{1}$ and $\mathrm{E}_{2}$; it arises as a result of that which exists at the time of occurrence of $\mathrm{E}_{1}$. In asserting (1) one makes an existential claim, namely that at the time of occurrence of $\mathrm{E}_{1}$ there exists that which can be completely described only by propositions which logically imply propositions which state that $\mathrm{E}_{2}$ occurs subsequently.

I assume here that Hume, in denying the possibility of the existence of logically necessary connections between events, would wish to deny the possibility of (1) being true, for any two successive events $\mathrm{E}_{1}$ and $\mathrm{E}_{2}$. In what follows I shall occasionally employ "There exists a necessary connection between $\mathrm{E}_{1}$ and $\mathrm{E}_{2} "$ as a shorthand expression for (1).

I turn now to a consideration of arguments, including Hume's, which may be deployed against the thesis that it is possible, conceivable, as far as we can ever know for certain, that (1) is true, for some pairs of successive events $\mathrm{E}_{1}$ and $\mathrm{E}_{2}$.

These arguments fall into three categories. In the first place it may be argued that (1) is meaningless. Secondly, it may be argued that (1) is analytically false, that it is not a conceptual possibility that (1) could be true. But finally it might be argued that (1) is analytically true. The point is that in asserting (1) of two events, $\mathrm{E}_{1}$ and $\mathrm{E}_{2}$, one is making an existential claim. If the thesis that it is possible that (1) is true is to amount to anything, then (1) must be interpreted in such a way that it is not true in any universe whatsoever, i.e. not analytically true.

One main argument employed by Hume can be dismissed without much discussion. Hume argues, essentially, that it cannot be meaningful to assert that there exists a necessary connection between two events $\mathrm{E}_{1}$ and $\mathrm{E}_{2}$ because there is no impression which could give rise to the idea of such a necessary connection. A basic tenet of Hume's Treatise is 'all our ideas are copied from our impressions'.

In somewhat more modern terminology this argument might be presented as follows:

(a) Only those words (sentences) which can be given a phenomenalistic analysis, i.e. which can be analysed in terms of words (sentences) that refer to sense data, are meaningful.

(b) 'Necessary connection' as employed in the sentence "There exists a necessary connection between $\mathrm{E}_{1}$ and $\mathrm{E}_{2}$ ' cannot be given such an analysis. Hence 'There exists a necessary connection between $\mathrm{E}_{1}$ and $\mathrm{E}_{2}$ ' cannot be meaningful.

I assume without discussion that no one today would wish to defend any such phenomenalistic criterion of meaning as (a) above.

Much of Hume's discussion concerning causation may be construed as a defence of the epistemological thesis that it is impossible to know with complete certainty that any particular observed event, which we describe perhaps as $\mathrm{E}_{1}$, will be followed by an event that we describe as $\mathrm{E}_{2}$. This epistemological thesis I accept without reservation: we must just admit that it is always possible, as far as we can ever know for certain, that things may suddenly cease to happen as we expect or predict. However this epistemological thesis does not imply that the ontological thesis "There exist necessary connections between successive events" (where this is understood as in (1)) is false. These two theses are in fact perfectly compatible. For suppose the ontological thesis is true. Suppose that at the time of occurrence of a particular observed event $E_{1}$ there exists that which can only be completely described by propositions $\mathrm{P}_{1}$ which logically imply a proposition $\mathrm{P}_{2}$ that states that $\mathrm{E}_{2}$ occurs subsequently. It is perfectly compatible with this to assert that at the time of occurrence of $\mathrm{E}_{1}$ it is impossible to know for certain that $E_{2}$ will occur subsequently, for of course it may well be impossible to know for certain that $\mathrm{P}_{1}$ is true.

1 would wish in fact to argue that there is no empirical statement that can be known to be true with complete certainty. I support, in other words, Einstein's dictum: 'In so far as 
propositions refer to reality they are not certain: in so far as they are certain they do not refer to reality. ${ }^{2}$

Briefly, then, the position defended here is this: $(a)$ we cannot be certain that $\mathrm{E}_{2}$, say, will follow a particular event we observe and describe perhaps as $\mathrm{E}_{1}$; but equally $(b)$ we cannot be certain that $\mathrm{E}_{2}$ does not necessarily follow $\mathrm{E}_{1}$ (in the sense of (1)).

Another argument, due to Hume, which may be deployed against the thesis that (1) is possibly true, runs, briefly, somewhat as follows. Given any two successive events, $\mathrm{E}_{1}$ and $\mathrm{E}_{2}$, it is always possible that $\mathrm{E}_{1}$ should not be followed by $\mathrm{E}_{2}$, possible in the sense that $(a)$ we can imagine that $\mathrm{E}_{1}$ is not followed by $\mathrm{E}_{2}$, or $(b)$ no contradiction is involved in asserting that $\mathrm{E}_{1}$ is not followed by $\mathrm{E}_{2}$. (In what follows the first criterion is ignored, since we are here concerned with logical necessity.) Since it is logically possible that $\mathrm{E}_{1}$ is not followed by $\mathrm{E}_{2}$, it cannot be logically necessary that $E_{1}$ is followed by $E_{2}$.

But is it always logically possible, given any two successive events $\mathrm{E}_{1}$ and $\mathrm{E}_{2}$, that $\mathrm{E}_{1}$ should not be followed by $\mathrm{E}_{2}$ ? Is the statement ' $\mathrm{E}_{1}$ is not followed by $\mathrm{E}_{2}$ ' invariably noncontradictory? Suppose $\mathrm{E}_{1}$, that which exists at the instant in question, is such that any complete description, or specification, of $\mathrm{E}_{1}$ logically implies propositions which state that $\mathrm{E}_{2}$ occurs subsequently. Suppose that the meaning of ' $\mathrm{E}_{1}$ ' is such that the statement ' $\mathrm{E}_{1}$ occurs' logically implies some such statement as ' $\mathrm{E}_{2}$ occurs subsequently'. In this case ' $\mathrm{E}_{1}$ is not followed by $\mathrm{E}_{2}$ ' is a logical contradiction. Of course no doubt if one gives only a certain kind of true description of the two events - a description in terms of what the two events look like, for example - so that $\mathrm{E}_{1}$ is described as ' $\mathrm{E}_{1}{ }^{*}, \mathrm{E}_{2}$ as ' $\mathrm{E}_{2}{ }^{*}$ ', let us say, then it may well be that ' $\mathrm{E}_{1} *$ is not followed by $\mathrm{E}_{2} *$ ' is invariably non-contradictory. But this result does not establish the inconceivability

Clearly in order to show that (1) cannot possibly be true it is necessary to show that that which exists at any instant cannot conceivably be such that only propositions which logically imply some proposition which states that such and such occurs subsequently, can completely state what it is that exists at the instant in question.

This thesis may be defended as follows. Given any two successive events, $E_{1}$ and $E_{2}$, if a proposition, $P_{1}$, which is intended to be a description of $E_{1}$, logically implies that $E_{2}$ follows $\mathrm{E}_{1}$ then ' $\mathrm{P}_{1}$ ' must include some such statement as 'This event is followed by $\mathrm{E}_{2}$ ' or ' $\mathrm{E}_{1}$ is followed by $\mathrm{E}_{2}$ '. But ' $\mathrm{E}_{1}$ is followed by $\mathrm{E}_{2}$ ' cannot be interpreted as referring exclusively to what exists at the time of occurrence of $E_{1}$. ' $E_{2}$ follows $E_{1}$ ' refers to a relational property of $E_{1}$; it can no more be said to refer exclusively to what exists at the time of occurrence of $E_{1}$ than say 'A is to the left of B' can be said to refer exclusively to what exists at the place where A is. Hence (1) cannot possibly be true of any events.

My reply to this argument is as follows. There are at least two distinct ways in which 'event', 'object' and 'change' may be conceived, two conceptual schemes, which I shall call $\mathrm{C}_{1}$ and $\mathrm{C}_{2}$. The above 'relational' argument against the possibility of (1) being true is valid granted $\mathrm{C}_{1}$, invalid granted $\mathrm{C}_{2}$.

I shall give here only a brief, informal description of $\mathrm{C}_{1}$ and $\mathrm{C}_{2}$.

$\mathrm{C}_{1}$ and $\mathrm{C}_{2}$ may be contrasted as follows. $\mathrm{C}_{1}$ is committed in the first instance to an ontology of 'events' or 'time-slices', statements about objects that persist and change being analysable in terms of, or reducible to, statements about successive 'events' or 'time-slices'. $\mathrm{C}_{2}$, on the other hand, is committed in the first instance to an ontology of persisting, changing objects, statements about instantaneous events being reducible to statements about persisting, changing objects.

According to $\mathrm{C}_{1}$ the universe is a sort of four-dimensional array of point-events. The ultimate entities of $\mathrm{C}_{1}$ are point-events, and the ultimate properties are properties that can be ascribed to point-events. Just as there are spatial relations between point-events, such ' $x$ is above $y$ ' so there are temporal relations between point-events, such as ' $x$ is earlier than y'. (Of course both 'above' and 'earlier than' presuppose conventionally assigned 'directions', which may however be suggested by certain pervasive features of the array of point-events.) Just as spatial relations are the essence of Space, so temporal relations are the essence of Time. 
Notions of 'object', 'persistence' and 'change' can be defined in terms of the above as follows. A homogeneous spatio-temporal array of point-events can be termed an 'object': objects are thus four-dimensional entities. If the object has a temporal length of $t$ units, then the object 'persists' for those $t$ units. If the point-events at one 'temporal end' of the object differ in a systematic way from the point-events at the other 'temporal end', then the object 'changes' in the interval $t$. At different moments or instants throughout $t$, there exist different temporal parts of the object just as different spatial parts exist at different places.

Granted this conceptual scheme one can, I think, legitimately argue that just as the spatial relation 'B is to the left of A' can scarcely be said to exist exclusively at the place where A exists, so the temporal relation ' $\mathrm{E}_{2}$ is later than $\mathrm{E}_{1}$ ' can scarcely be said to exist exclusively at the time of occurrence of $\mathrm{E}_{1}$.

According to $\mathrm{C}_{2}$ notions of 'persistence' and 'change' are of the essence of time, and are not derivable notions. Each presupposes the other. If something persists then something must change, for if there was no change, nothing would happen, there would be no 'passage of time', and hence no persistence. Again, if a thing changes then that thing must persist throughout the change. And even if it is simply the case that there are changes, without there being any one thing which suffers these changes, nevertheless a place must be identified throughout the changes, and this requires the persistence of something.

The ultimate entities of $\mathrm{C}_{2}$ are objects which, by definition, persist and change. Ultimate properties of $\mathrm{C}_{2}$, which are ascribable to objects, presuppose the notions of persistence and change. A description of an instantaneous event states what is true of objects at that instant; such a description thus presupposes the notions of persistence and change. In other words, whereas, in $\mathrm{C}_{1}$, statements about persisting, changing objects presuppose statements about successive instantaneous events that do not involve the notions of 'persistence' and 'change', in $\mathrm{C}_{2}$, statements about instantaneous events presuppose statements about persisting changing objects.

According to $\mathrm{C}_{2}$, objects have spatial parts, but no temporal parts. Objects are three-dimensional, not four-dimensional, entities. Certainly objects persist and change; but to say this is not to say that objects are composed of their successive instantaneous states. It is not an object, but rather the life-history of an object, that is composed of the successive states of that object. And an object is not to be identified with its life-history.

It is, I think, clear that the ordinary commonsense way of conceiving the world presupposes $\mathrm{C}_{2}$ rather than $C_{1}$. Physical things - tables, stones, birds, etc.-are clearly 'objects' in the sense of $C_{2}$ rather than $\mathrm{C}_{1}$. 'Object' in the sense of $\mathrm{C}_{1}$ we should ordinarily translate as 'life-history of an object'. Instead of thinking of an object as a spatio-temporal array of events (which presupposes $\mathrm{C}_{1}$ ), we ordinarily think of an event as the instantaneous state of a number of persisting, changing objects (which presupposes $\mathrm{C}_{2}$ ).

We tend however to think of 'Time' in spatial terms. This is perfectly compatible with $\mathrm{C}_{2}$ if it involves no more than simply thinking of persistence/change, i.e. time, in metrical terms. But if it involves thinking of objects that persist and change as being extended in time, as being fourdimensional entities made up of temporal parts, then clearly thinking of time in spatial terms is completely inconsistent with $\mathrm{C}_{2}$. The fact that we do tend to 'spatialize' time in this second sense explains much of our ordinary puzzlement about the nature of Time. Any picture of Time which has the Present moving up an extended time-line constitutes a hopeless, contradictory fusion of $\mathrm{C}_{1}$ and $\mathrm{C}_{2}$.

If one accepts $\mathrm{C}_{2}$, the above 'relational' argument against the possible existence of necessary connections between successive events, or states of affairs, is no longer valid, since the analogy between temporal and spatial relations collapses. I assume here that the world can legitimately be conceived in terms of $\mathrm{C}_{2}$, and that physical theories can be formulated in terms of $\mathrm{C}_{2}{ }^{3}$

It may be objected: But if there is to be any change in the world, objects must have different properties, or be in different states, at different times. Now suppose an object, $X$, is in the state $S_{1}$ at time $t, S_{2}$ at time $t+d t$. Then any description of what exists at time $t$, which logically implies ' $X$ is $S_{2}$ at time $\mathrm{t}+\mathrm{dt}$ ', must incorporate some such statement as ' $\mathrm{X}$ is $\varphi$ at time $t$ ', where the meaning of $\varphi$ is 
such that an object is $\varphi$ at time $t$ only if it is $S_{1}$ at time $t$ and $S_{2}$ at time $t+d t$. But here $\varphi$-ness is not the sort of property that can conceivably exist at an instant, that an object can con ceivably have at an instant. That which exists at time $t$, and is described by ' $\mathrm{X}$ is $\varphi$ at time $\mathrm{t}$ ' is also described by ' $\mathrm{X}$ is $\mathrm{S}_{1}$ at time t'. And this latter description does not logically imply ' $X$ is $S_{2}$ at time $t+d t$ '. Hence it is not the case that which exists at time $\mathrm{t}$ is such that any description of this logically implies ' $\mathrm{X}$ is $S_{2}$ at time $\mathrm{t}+$ $\mathrm{dt}^{\prime}$. In other words, even if $C_{2}$ is presupposed, (1) above cannot conceivably be true.

My answer to this argument is as follows. The above argument assumes that any statements of the form ' $\mathrm{X}$ is $\varphi$ at time $\mathrm{t}$ ', where these entail some statement about how $\mathrm{X}$ is, or is not, changing at time $\mathrm{t}$, can invariably be reduced to a set of statements of the form ' $X$ is $S_{0}$ at time $t^{\prime}$, ' $X$ is $S_{1}$ at time $t_{1}$ ', ' $X$ is $S_{2}$ at time $t_{2}{ }^{\prime} \ldots$ where $t<t_{1}<t_{2}<\ldots$, and where not one of these statements, taken on its own, entails any statement about how $\mathrm{X}$ is, or is not, changing at the instant in question. The above argument assumes, in other words (to state the thing a little more generally), that any statement about an object that involves the notions of' change' or 'persistence', can be reduced to, or translated into, a set of statements that attribute similar and dissimilar properties to the object at different times, where none of these statements, taken individually, involves the notions of 'change' or 'persistence'. But it is precisely this assumption which must be rejected if $C_{2}$ is accepted or presupposed. Within $\mathrm{C}_{2}$, there are predicates applicable to objects which presuppose the notions of 'persistence' and 'change', and which cannot be reduced to predicates which do not presuppose these notions. The thesis that there are such predicates is absolutely essential to $C_{2}$. For the basic entity of $C_{2}$ is the 'object', which, by definition, 'persists' and 'changes'; and to say this is to say that to any object of $\mathrm{C}_{2}$ a predicate can be applied which presupposes the notions of 'persistence' and 'change', and which cannot be reduced to predicates which do not presuppose these two notions.

At this point one might almost be tempted to conclude: If $\mathrm{C}_{2}$ is incompatible with the above assumption, with the assumption, that is, that any predicates involving the notions of persistence and change can be reduced to predicates that do not involve these notions, then so much the worse for $C_{2}$. However, in the next section I shall defend a thesis, which I shall call 'dispositional realism', which entails that the above assumption is false.

One last remark about the distinction between $\mathrm{C}_{1}$ and $\mathrm{C}_{2}$. It has sometimes been suggested that the question of whether one accepts a basic ontology of 'events', or of 'changing persisting things' (i.e. whether one accepts $\mathrm{C}_{1}$ or $\mathrm{C}_{2}$ ), depends on, or is related to, the question of whether one's basic language is tenseless or tensed. ${ }^{4}$ The two questions are however entirely distinct. How one answers the question: Are propositions basically tensed or tenseless? depends simply on what one chooses to mean by the term 'proposition'. If one chooses to employ the term 'proposition' in such a way that a proposition can, with the passage of time, change its truth-value, then tense will indeed be basic and uneliminatable. According to this use of 'proposition', 'Harold Wilson is talking', for example, expresses, at different times, the same proposition: thus one and the same proposition can be true at one time, false at some other time. Attempts to eliminate the tense of 'Harold Wilson is talking' must fail, for such attempts will produce sentences of the form 'Harold Wilson is (tenseless) talking at time t'; and this sentence expresses different propositions at different times, granted the above interpretation of the term 'proposition'.

One may, however, of course employ the term 'proposition' in such a way that propositions do not change their truth-value with the passage of time. In this case the sentence 'Harold Wilson is (tenseless) talking at time t' expresses the same proposition at all times (granted t is some definite time), whereas 'Harold Wilson is (tensed) talking' expresses different propositions at different times. In this case tense can be eliminated simply by reformulating any tensed sentence so that the time to which the sentence refers does not depend on the time at which the sentence is uttered.

All this has nothing whatsoever to do with whether one accepts $\mathrm{C}_{1}$ or $\mathbf{C}_{2}$. For one accepts $\mathrm{C}_{1}$ if and only if one accepts that all predicates which presuppose the notions of persistence and change can be reduced to predicates which do not presuppose these notions. And one accepts $\mathrm{C}_{2}$ if and only if one rejects the above thesis.

The temptation to associate $\mathrm{C}_{2}$ and $\mathrm{C}_{\mathrm{t}}$ with the above two theses about tensed propositions might arise in the following kind of way. On the one hand it is tempting to think that if the world consists, 
in the first instance, of persisting, changing objects, then, in the first instance, one's propositions must also persist and change, i.e. must change their time-reference and truth-value with the passage of time. But a consequence of employing the term 'proposition' in this kind of way is that tense cannot be eliminated from propositions. Thus $\mathrm{C}_{2}$ becomes associated (for wholly fallacious reasons) with the thesis that tense cannot be eliminated. On the other hand it is also tempting to think that if one employs the term 'proposition' in such a way that propositions do not change their time-reference and truth-value with the passage of time (so that tense can be eliminated), then one is committed in the first instance to an ontology of 'events', or 'time-slices'. In this way the thesis that tenses can be eliminated becomes associated with $\mathrm{C}_{1}$.

\section{II}

So far I have been arguing against objections to the thesis that it is conceivable that (1) is true, for some two events $E_{1}$ and $E_{2}$. In a sense, therefore, I have not yet said anything directly in support of the conceivability of (1). There remains the problem: Is it conceivable that that which exists at some instant is such that it can only be completely described by propositions which logically imply that some event occurs subsequently? In order to answer this question it will be convenient to examine whether or not the kind of descriptions we ordinarily give of things, and the descriptions of physics, could ever logically imply descriptions of subsequent states of affairs. Ultimately I hope to establish the conceivability of (1) by establishing that:

(a) It is conceivable that a certain type of realistic, comprehensive, deterministic physical theory is true.

(b) If a theory of this type is true, then (1) is true for successive states of affairs $E_{1}$ and $E_{2}$.

Although the ordinary descriptions we give of things do not logically imply descriptions of subsequent states of affairs, nevertheless such descriptions do carry implications about how the things described change. In describing something as 'solid', 'inflammable', 'soluble', 'magnetic', 'elastic', 'hot', 'heavy', 'soft', 'sticky', or 'brittle', for example, we not only attribute a property to that thing, but we also imply that the thing will, in certain circumstances, 'behave' or 'change' in certain definite ways. This applies also to such descriptions as 'stone', 'copper', 'water', 'salt', 'glass', 'petrol', 'oxygen', etc., in that such substances have certain properties, of the above type, by definition.

The point to note is this: It is not just a contingent matter of fact that, for example, solid objects are objects which do not pass effortlessly through each other. It is implicit in the meaning of the term 'solid' that one solid object does not pass effortlessly through another solid object. Similarly it is implicit in the meaning of the term 'inflammable' that an object which is inflammable, and which is exposed to a naked flame, itself bursts into flames. In other words, the meaning of the term 'inflammable' is such that ' $\mathrm{X}$ is inflammable at time /' and ' $\mathrm{X}$ is exposed to a naked flame at time $t$ ' taken together, logically imply 'X bursts into flame at time $t$ '. Similar considerations arise in connection with the meaning of the other terms mentioned above, 'soluble', 'magnetic', 'elastic', etc.

There is, however, a very simple reason why statements of the above type, which attribute the above type of properties to objects at some instant, cannot logically imply statements which attribute properties to the objects at some subsequent instant. The reason is this: It is always a logical possibility that any object which possesses any property of the above type might suddenly and inexplicably cease to possess this property. A liquid that is inflammable at one instant might suddenly cease to be inflammable at a subsequent instant: a solid object might suddenly melt, evaporate, explode or vanish.

Of course in giving the above kind of descriptions of things we ordinarily presuppose that objects will not inexplicably vanish, petrol inexplicably cease to be inflammable, etc.; we presuppose 
that, in general, the above kind of properties will change only in the customary known ways. In fact a sort of general inter-related stability in the way in which properties persist and change is essential if the kind of descriptions we ordinarily give of things is to have any application at all. Inflammability, solidity, elasticity, etc., constitute properties that objects either do or do not possess only in the context of the hypothesis that these properties in general change and persist only in certain constant, inter-related ways. It is only because it is presupposed that in general an object only becomes or ceases to be inflammable under certain specific circumstances (e.g. when soaked in petrol or water) that an assertion such as 'X is inflammable at time t', where $\mathrm{X}$ is not exposed to a naked flame at time $t$, qualifies as a description of what exists at that instant. If nothing can be presupposed about the way in which the inflammability of an object does and does not change, then it would make, in a sense, no difference whatsoever whether one ascribed inflammability or non-inflammability to an object at a moment when it was not exposed to a naked flame, for whatever happened subsequently when the object was exposed to a naked flame, one could always argue that the object had become, remained, or ceased to be inflammable (as the case might be) in the intervening time.

The fact remains, however, that there is no precise, exceptionless, comprehensive hypothesis about the way in which such properties as inflammability, solidity, elasticity, etc., do and do not change incorporated explicitly in the meaning of the words 'inflammable', 'solid', 'elastic', etc. It is essentially for this reason that the ordinary descriptions we give of things cannot logically imply descriptions of subsequent states of affairs.

Nevertheless, the following at least can be maintained: The ordinary descriptions we give of things, the ordinary, commonsense way in which we conceive the world, presuppose that:

(2) There are irreducible properties, possessed by objects at an instant, which are such that any statement which attributes such a property to an object at a time $t$ will, when combined with some further statement which merely states something about the state of affairs at time $t$, logically imply a statement about how the object is changing at time $t$.

I should perhaps add that this does not mean that any attribution of a 'disposition' to something constitutes ascribing a property to that thing. In saying of someone that he is a cigarette-smoker, for example, one says just that the person smokes cigarettes from time to time. It is not logically possible for a person to be a cigarette-smoker and never smoke a cigarette. ' $\mathrm{X}$ is inflammable', on the other hand, does not mean ' $\mathrm{X}$ bursts into flames from time to time'. An object may be inflammable even though it never bursts into flames.

I must now consider two possible objections to the claim that the ordinary descriptions we give of things presuppose thesis (2), or 'dispositional realism' as this thesis might be called.

In the first place it may be objected: An object or substance which is inflammable may not burst into flames when exposed to a naked flame for any number of different reasons: There may not be enough oxygen present; the object may be slightly damp; the flame may not be hot enough, etc. Hence the meaning of 'inflammable' cannot be such that ' $\mathrm{X}$ is inflammable at time $\mathrm{t}$ ' and ' $\mathrm{X}$ is exposed to a naked flame at time $t^{\prime}$ logically imply 'X bursts into flames at time $t$ '. Similar considerations arise in connection with all the other 'dispositional properties'-solidity, elasticity, etc.

But this argument simply exploits a certain vagueness in the meaning of 'inflammable'. In saying that an object is inflammable one might mean anything from 'If this object is heated to $6,000^{\circ} \mathrm{C}$. in an atmosphere of pure oxygen, after an hour or so the object will show slight signs of oxidation', to 'The object ignites spontaneously at room temperature when exposed to the air'. In other words, the above objection no longer applies once a precise meaning has been assigned to 'inflammable'.

In the second place it may be objected: To ascribe inflammability or solidity, etc., to an object at some instant is not to ascribe a property to the object at that instant. Objects burst into flames or fail to ignite: but at those moments when they are not exposed to naked flames, objects do not possess some mysterious additional property of inflammability or non-inflammability. To assert 'X was inflammable at time $\mathrm{t}$ ', assuming $\mathrm{X}$ was not exposed to a naked flame at time $\mathrm{t}$, is to assert no more than 'If $\mathrm{X}$ had been exposed to a naked flame at time $t, \mathrm{X}$ would have burst into flames'. In 
this manner any reference to a property such as inflammability, solidity, elasticity, etc., can be eliminated. Thus despite appearances to the contrary, the statement ' $\mathrm{X}$ is inflammable' does not really attribute any property to $X$.

I shall now attempt to refute this thesis.

The first point to note is that the thesis leaves utterly obscure what is to count as an ascription of a property to an object at an instant For presumably any statement that 'supports a counterfactual conditional', i.e. implies a statement of the form 'If such and such were the case, then such and such would happen', cannot, if the above thesis is accepted, be construed as a straightforward description of what exists. But any ordinary description of things, events, states of affairs, in the world, will support 'counter-factual conditionals'. Bereft of such statements as ' $\mathrm{X}$ is solid', ' $\mathrm{X}$ is heavy', ' $\mathrm{X}$ is hot', ' $\mathrm{X}$ is fluid", ' $\mathrm{X}$ is soft', etc., and 'X consists of water', ' $\mathrm{X}$ consists of copper', etc., one is left entirely in the dark as to what precisely can be attributed to objects at any given instant.

Here then is a major objection to the thesis that reference to dispositions] properties can be eliminated. Interpreting ' $\mathrm{X}$ is inflammable at time t' to mean 'If $\mathrm{X}$ were exposed to a naked flame at time $t, X$ would burst into flames at time $t$ ' does not help to eliminate reference to dispositional properties, because 'is exposed to a naked flame' and 'bursts into flames' are just as dispositional as 'is inflammable'. For example, the sudden appearance of a wavering light about an object does not mean that that object has burst into flames if the object is in no way changed or consumed with the passage of time. ${ }^{6}$

One obvious reply to this objection is that only those statements which ascribe properties to sense-data can qualify as irreducible descriptions of what exists at an instant. But this thesis is equivalent to phenomenalism, and there are well-known objections to phenomenalism, which I shall not discuss here. ${ }^{7}$

Granted that phenomenalism is untenable, the thesis that the ordinary descriptions we give of things do not presuppose (2) must, it seems, be rejected.

But in any case, what possible reason can there be for holding that statements that incorporate dispositional terms should be reducible to statements that incorporate only nondispositional terms? Why should it be thought desirable to be able to analyse any dispositional terms such as 'inflammable' in terms of purely non-dispositional terms?

One answer to this question might of course be the following: The basic descriptive terms should be such that it is always possible to verify or falsify conclusively whether a particular description is true of a particular object. But any description that incorporates a dispositional term cannot always be conclusively verified or falsified. ' $\mathrm{X}$ is inflammable', or ' $\mathrm{X}$ is solid', for example, cannot be conclusively verified or falsified at those times when $\mathrm{X}$ is not being tested for inflammability.

The simple answer to this is, of course, that no descriptions, however 'epistemologically basic', can be conclusively verified or falsified. Even on those occasions when $\mathrm{X}$ is being tested for inflammability or solidity, one still cannot conclusively verify or falsify ' $\mathrm{X}$ is inflammable' or ' $\mathrm{X}$ is solid', for this would require the conclusive verification of certain other descriptive statements which would in turn incorporate dispositional terms. It is only when certain low-level assumptions have been made about how relevant dispositional properties are correlated and conserved - assumptions presupposed by such 'basic' statements as ' $\mathrm{X}$ is a material object', ' $\mathrm{X}$ is water', ' $\mathrm{X}$ is copper', etc.- - that any singular description such as ' $\mathrm{X}$ is inflammable at time $\mathrm{t}$ ' can be verified or falsified. Assumptions about how dispositional properties are correlated and conserved can of course be tested individually: they cannot however be tested en masse, for then the whole machinery of testing would break down. No observation at one instant would have any relevance to any observation at any other instant, and the fully fledged Humean nightmare would be upon one.

Of course if one wishes to defend phenomenalism, if, in other words, one accepts a sort of updated version of Hume's thesis 'All our ideas are copied from our impressions', then the meaning of terms such as 'inflammable' will seem wholly obscure unless they can be defined phenomenalistically, and hence non-dispositionally. But once phenomenalism, and the above criterion of 
meaning, have been rejected, there seems to be no very good reason why it should be thought to be desirable to define dispositional predicates in terms of non-dispositional predicates.

There is perhaps one other reason why it might be felt that dispositional predicates require analysis in terms of non-dispositional predicates if they are to have any clear meaning. Dispositional predicates clearly presuppose the notions of persistence and change. From statements that attribute dispositions] properties to objects at some time t one can deduce statements about how some object is changing at that instant. Further, it is only in the context of assumptions about how dispositional properties are correlated and conserved-i.e. in the context of assumptions about how dispositional properties persist and change - that it becomes meaningful to attribute dispositional properties to objects at all. It may be assumed however that any statement which ostensibly attributes a property to an object at some instant, but which presupposes the notions of persistence and change, ought to be analysable in terms of statements which attribute similar and dissimilar properties to the object at successive instants, where none of these statements, taken individually, involves the notions of persistence and change. But, as I remarked in the previous section, it is precisely this assumption which must be rejected if $\mathrm{C}_{2}$ is accepted. In other words, if $\mathrm{C}_{2}$ is accepted, one has no cause whatsoever to be disturbed by the fact that the ultimate properties of things are dispositional properties.

Despite the undesirability and impossibility of reducing dispositional terms to non-dispositional terms, various philosophers have persisted in the attempt. And as a result, what requires analysis is not so much the meaning of 'inflammable' etc. but rather the meaning of terms coined by philosophers in their attempts to analyse 'inflammable' etc.

In the first place, the whole 'problem' of 'subjunctive conditionals' or 'contrary-to-fact conditionals' or 'counter-factual conditionals' seems to have arisen simply as a result of a refusal to countenance the idea that, for example, ' $\mathrm{X}$ is inflammable at time $\mathrm{t}$ ' might ascribe a property to $\mathrm{X}$ at time $\mathrm{t}$, where the meaning of 'inflammable' is such that the above statement, together with ' $\mathrm{X}$ is exposed to a naked flame at time $t$ ', logically implies 'X bursts into flames at time t'. For if one insists that ' $\mathrm{X}$ was inflammable at time $\mathrm{t}$ ' must be interpreted as 'If $\mathrm{X}$ had been exposed to a naked flame at time $t, X$ would have burst into flames at time $t$ ', one then creates the problem of how this latter statement is to be analysed.

Some philosophers have argued that this so-called 'problem of counter-factual conditionals' is to be solved along the following lines: a counter-factual conditional makes an implicit appeal to a universal conditional, where this is to be understood as asserting 'nomic' as opposed to 'accidental' universality. According to this kind of view a statement asserts 'nomic' universality if it is a universal statement that meets certain 'logical and epistemic requirements'.

But according to the position developed here, this sort of approach puts the cart before the horse. For, according to the view defended here, the meaning of a counterfactual conditional such as 'If this object had been exposed to a flame at time t, it would have burst into flames', at least under one legitimate interpretation, poses no problem whatsoever; the statement means simply 'This object was inflammable at time $\mathrm{t}$ (and was not exposed to a naked flame at time t)'. Hence, if the

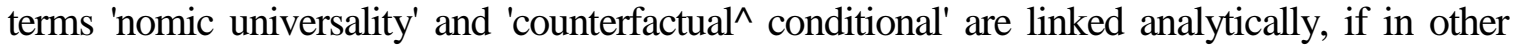
words 'A statement that asserts nomic universality supports counterfactual conditionals' is analytic, then 'nomic universality' should be analysed in terms of 'counterfactual conditional' and not the other way round. This can be done very simply as follows. Consider for example the following statement:

(3) All the screws in Smith's car are rusty.

If this statement is interpreted as asserting 'accidental' universality only, it can be written as:

(4) (x) ((x is a screw \& $x$ is in Smith's car) $\supset x$ is rusty).

If (3) is interpreted as asserting 'nomic' universality, then it can be written as:

$(5)(x)(x$ is a screw $\supset x$ is $R)$ 
where ' $\mathrm{R}$ ' is a predicate such that ' $x$ is R at time $t^{\prime}$ and ' $x$ is in Smith's car at time t' together logically imply ' $x$ is rusty at time $\mathrm{t}$ '. (Here R-ability is a property analogous to inflammability or instability: 'R-able' and 'rustable' differ of course because, roughly speaking, it is exposure to Smith's car, not to air and water, that rusts an R-able object.)

It is I hope clear that (5) asserts 'nomic' universality because it supports the counterfactual 'If this screw were in Smith's car at time $t$ it would be rusty at time $t$ ', where this means simply "This screw is R'. (4) on the other hand asserts only 'accidental' universality because it does not support this counterfactual conditional. Any assertion of (3) would of course be interpreted as asserting only 'accidental' universality, simply because no one wishes to attribute the property $\mathrm{R}$ to screws.

This interpretation of the term 'nomic' universality is not put forward as the only conceivable interpretation: it is put forward simply as a legitimate interpretation, granted that there is the above analytic tie-up between 'counterfactual conditional' and 'nomic universality'. In particular it must be stressed that the phrase 'asserts nomic universality', as employed here, is not intended to be equivalent to 'is a law-like statement', or 'asserts a physical law'. Clearly a statement that asserts nomic universality must satisfy further conditions before it can be called a physical law: one might, for example, stipulate that a physical law can contain no reference, explicit or implicit, to a particular object or a particular spatio-temporal region.

It may, however, be held that any reasonable interpretation of 'nomic' universality must be such that asserting nomic universality constitutes, if not a sufficient, then at least a necessary condition for a statement to be a physical law. And, it may be held, there are many universal statements which we wish to regard as statements of physical laws, which do not assert 'nomic' universality in the above sense.

Consider, for example, Newton's law of gravitation. If this asserts 'nomic' universality then it supports the following kind of counterfactual conditional:

(6) If this object had been over there during the interval $\Delta \mathrm{t}$, then it would have moved in accordance with Newton's law.

But this statement, according to the above interpretation, is equivalent to attributing a dispositional property — gravitational charge - to the body during the interval $\Delta \mathrm{t}$ in question. Hence Newton's law of gravitation can only be interpreted as asserting 'nomic' universality if it is interpreted as attributing the dispositional property of gravitational charge (proportional to mass) to all bodies. But this interpretation amounts to the kind of 'essentialist' interpretation defended for example by Cotes, but rejected by Newton himself. And although Newton rejected this interpretation, he clearly did not regard the law of gravitation as asserting 'accidental' universality only. How then can one interpret a law as asserting 'nomic' universality, in the above sense, if the law is not given an 'essentialist' interpretation, i.e. if it is not interpreted as ascribing a dispositional property to objects?

The answer to this question is, I suggest, as follows: a law which is not, for one reason or another, given the above type of 'essentialist' interpretation may nevertheless justifiably be interpreted as asserting 'nomic' universality, in the above sense, as long as it is held to be conceivable that there is an essentialist law which entails or replaces the first law. Newton, for example, did not reject essentialism as such; he merely rejected as unacceptable the dispositional property of gravitational charge. Any theory which entailed or replaced the law of gravity, and which attributed dispositional properties to corpuscles which did not involve the notion of 'action at a distance', and which supported the counterfactual (6), would have been, it may be presumed, entirely acceptable to Newton.

To this it may be objected: But to insist that physical laws should assert 'nomic' universality in this sense is to commit physics to 'essentialism' in an important sense, even if not all acceptable physical laws are required to be essentialistic. But there are overwhelming objections to committing physics to 'essentialism' in this way. Hence physical laws cannot be required to assert 'nomic' universality in the above sense. 
In reply to this objection I shall make just two comments.

(a) If there are overwhelming objections to committing physics to 'essentialism', then what the above discussion has shown is that in a perfectly straightforward, entirely legitimate, sense of 'nomic universality', physical laws do not assert nomic universality. ${ }^{10}$

(b) However, in the next section I shall in effect argue that there are no general objections to committing physics to an essentialistic programme.

\section{III}

In the last section I argued that statements that attribute dispositional properties to objects at some time t can logically imply statements about how some object is changing at time t. Further, I argued that it is only in the context of assumptions about how such dispositional properties are correlated and conserved-i.e. in the context of assumptions about how such dispositional properties persist and change - that it becomes meaningful to attribute dispositional properties to objects at all. It was maintained however that ordinary descriptions of objects at some instant cannot logically imply descriptions of subsequent states of affairs because there are no precise, comprehensive, exceptionless assumptions about how dispositional properties persist and change incorporated explicitly in the meaning of the terms which refer to these properties. Thus statements which ascribe inflammability, solidity, etc., to objects cannot logically imply statements about subsequent states of affairs because it is always logically possible that the objects might suddenly and inexplicably cease to be inflammable, solid, etc.

This suggests that in order to arrive at propositions which both qualify as descriptions of what exists at one instant and logically imply descriptions of what exist at subsequent instants, it will be sufficient to formulate a precise, comprehensive hypothesis about how things do and do not change, a comprehensive deterministic physical theory, in other words, which is explicitly incorporated in, or presupposed by, the meaning of the terms of the hypothesis in question.

More precisely, I suggest that in order to establish the conceivability of (1), it will be sufficient to establish that it is conceivable that that which exists at some instant is such that only propositions which incorporate terms whose meanings presuppose the truth of some comprehensive deterministic physical theory, can completely describe that which exists at the instant in question.

No one denies of course that it is conceivable that propositions, P say, which specify (a) relevant deterministic physical laws, (b) so-called initial conditions, at some instant, can logically imply propositions, $\mathrm{P}_{2}$, which describe some subsequent state of affairs. But to acknowledge the conceivability of this is not to acknowledge the conceivability of (1), for of course the propositions $\mathrm{P}$ merely incorporate a description of what exists at the instant in question. The propositions $\mathrm{P}$ specify, not just the initial conditions, but also physical laws. In order to establish that (1) is conceivable, it is necessary to establish that it is conceivable that propositions, $\mathrm{P}_{1}$, which specify or describe no more than the initial conditions can logically imply propositions, $\mathrm{P}_{2}$, which describe some subsequent state of affairs. Or, more precisely, in order to establish that (1) is conceivable, it is necessary to establish that it is conceivable that that which exists at some instant - the initial conditions - is such that only propositions which incorporate terms whose meanings presuppose the truth of a comprehensive, deterministic physical theory can completely describe that which exists at the instant in question.

In order to clarify precisely what it is that I am proposing here, let us suppose that the world consists entirely of spherical, perfectly elastic particles, which interact by means of three kinds of forces, gravitational, 'electrical', and elastical. Suppose that the following somewhat crude Newtonian, atomistic, comprehensive physical theory, $\mathrm{T}$, is true. The postulates of $\mathrm{T}$ are as follows:

(i) If a material has elasticity $\mathrm{E}$ (with Young's modulus $=\mathrm{Y}$, Poisson's ratio $=\sigma, \mathrm{Y}$ and $\sigma$ being specific constants), then: 
(a) $\mathrm{F}=\underline{\mathrm{Y} . \Delta \mathrm{L} . \mathrm{A}}$ where $\mathrm{L}$ is the unstressed length of a rectangular rod of the material, $\Delta \mathrm{L}$ is the $\mathrm{L}$

increase in length corresponding to a force $\mathrm{F}, \mathrm{A}=$ unstressed area of cross-section of the rod, and $\mathrm{Y}=$ Young's modulus.

(b) $\Delta \mathrm{w}=-\underline{\sigma} . \Delta \mathrm{L}$ where $\mathrm{w}$ and $\mathrm{L}$ are the unstressed width and length of a rectangular rod of the $\mathrm{L}$

material, $\Delta \mathrm{w}$ is the decrease in width corresponding to an increase in length $\Delta \mathrm{L}$, and $\sigma=$ Poisson's ratio.

(ii) For any two particles with charges $\pm q_{1}$ and $\pm q_{2}, F=\frac{\left( \pm q_{1}\right) \cdot\left( \pm q_{2}\right)}{d^{2}}$.

(iii) For any two particles with masses $m_{1}$ and $m_{2}, F=\frac{G \cdot m_{1}}{d^{2}} \cdot m_{2}$.

(iv) $\mathbf{F}=$ m.a .

(v) If two forces $\mathbf{F}_{1}$ and $\mathbf{F}_{2}$ act on a body, then the resultant force $\mathbf{F}=\mathbf{F}_{1}+\mathbf{F}_{2}$.

(vi) Any particle of mass $\mathrm{m}$, charge $\pm \mathrm{q}$, unstressed radius $r$, elasticity $\mathrm{E}$, interacts with any other particle with mass $\mathrm{m}$, charge $\pm \mathrm{q}$, unstressed radius $\mathrm{r}$, elasticity $\mathrm{E}$ in such a way that $\mathrm{m}, \pm \mathrm{q}, \mathrm{r}, \mathrm{E}$ remain constant.

(vii) The world consists entirely of particles mass $m$, charge $\pm q$, unstressed radius $r$, elasticity $E$.

Granted that $\mathrm{T}$ is true, then propositions, formulated in terms of $\mathrm{T}$, which specify initial conditions, which describe what exists at any instant, will be of the following type: 'At $x, y, z, t$, there is a particle, mass $\mathrm{m}$, charge $+q$, elasticity $\mathrm{E}$, velocity $\mathrm{v}$, which is a sphere of radius $r$ when unstressed, but whose present shape and size is such and such'. Now suppose that that which exists at some instant is such that it can only be completely described if the descriptive terms employed, e.g. 'E', 'm', 'q' presuppose the truth of the postulates (i) - (vi) of T. Suppose, in other words, that that which exists at some instant is such that it can only be completely described in terms of $\mathrm{T}$ if the meaning of the terms ' $E$ ', ' $m$ ', ' 'q' are interpreted in such a way that the postulates (i) - (vi) of T are analytic statements. In this case a complete description of what exists at some instant, formulated in the terms of T, will presuppose the truth of postulates (i) - (vi) of T, and will thus logically imply descriptions of subsequent states of affairs. In other words, that which exists at some instant will be such that any set of propositions which gives a complete description of this will logically imply propositions which describe subsequent states of affairs.

It should be noted that the fact that the postulates (i) - (vi) of $\mathrm{T}$ are analytic does not mean that $\mathrm{T}$ itself is analytic, for of course (vii) is non-analytic. The entire empirical content of $\mathrm{T}$ is contained in the postulate (vii). (In what follows ' $\mathrm{T}$ ' is to be understood in such a way that the postulates (i) - (vi) are analytic statements.)

Terms of T such as 'mass', 'elasticity',' "electrical" charge', and 'gravitational charge' are intended to be analogous to such ordinary observational terms as 'inflammability', 'solidity', 'solubility', etc., in that they are intended as descriptive terms, as terms that can be used to ascribe a property to an object, to describe what exists at some instant. Of course a T-term differs from any ordinary observational term in that the meaning of a T-term presupposes the truth of a precise, comprehensive theory, whereas the meaning of a term such as 'inflammability' presupposes the truth of only some extremely limited and imprecise theory. I wish to argue, however, that this difference does not justify one in maintaining that only observational terms, and not also highly theoretical terms such as those of $\mathrm{T}$, can be descriptive, in the above sense.

I must now consider some objections to the thesis that (a) a physical theory such as $\mathrm{T}$ is conceivable, and (6) if such a physical theory is conceivable, then (1) is conceivable, or possibly true.

In the first place it may be argued that a physical theory that ostensibly postulates and describes certain unobservable, theoretical entities cannot be interpreted as really postulating and describing these entities, at least in so far as the theory is considered as a theory of physics. The job of a 
physical theory is to predict observable results of experiments, and this becomes possible only when the theoretical terms of a theory have been related to, or interpreted in terms of, observational terms by means of 'correspondence rules'. Thus a physical theory is 'about', not the theoretical entities it ostensibly postulates and describes, but rather the observable results that it predicts. Hence propositions, formulated in terms of the uninterpreted theoretical terms of a physical theory, that ostensibly describe initial conditions, cannot be regarded as really describing anything at all. Only propositions formulated in terms of observational terms can be interpreted as describing initial conditions, i.e. something that does actually exist.

There is not space for me here to give a reply to this objection. I shall assume, without discussion, that there is no valid, general objection to giving a tentatively realistic interpretation to physical theories. ${ }^{11}$ I shall assume that a physical theory such as T, formulated entirely in 'theoretical' terms, unrelated to any 'observational' terms by means of 'bridge' statements, or 'correspondence rules', can legitimately be interpreted as really postulating and describing the entities that it ostensibly postulates and describes. I assume, in other words, that 'theoretical' terms of physics, in addition to 'observational' terms, can be interpreted as descriptive terms, as terms intended to refer to entities or properties conjectured to exist. ${ }^{12} \mathrm{On}$ this view, 'bridge' statements, connecting 'observational' and 'theoretical' terms, constitute synthetic identity statements: they assert that certain observational entities or properties are, as a matter of contingent fact, certain theoretical entities or properties. ${ }^{13}$

Granted that there are no valid, general objections to interpreting a physical theory such as $\mathrm{T}$ realistically, it may nevertheless be objected that it is not conceivable that the particles, postulated by $\mathrm{T}$, could have, at any given instant, the kind of properties attributed to them by T. At most, it is conceivable that these particles could have, at any given instant, properties attributed to them by a theory $\mathrm{T}$ ', where $\mathrm{T}$ ' is similar to $\mathrm{T}$ in every respect except that at least some of the postulates (i)-(vi) are synthetic, not analytic. But propositions, formulated in terms of ' $\mathrm{T}$ ', that describe initial conditions, that state what exists at some instant, cannot alone logically imply descriptions of subsequent states of affairs. Hence even if it is granted that it is conceivable that a true, realistically interpreted, deterministic, comprehensive physical theory can be formulated, this does not guarantee that (1) is conceivably true.

My reply to this objection is as follows. If $\mathrm{T}$ ' is interpreted realistically then it follows analytically, from what one means by 'realistic interpretation', that at least some of the postulates of $\mathrm{T}^{\prime}$ are analytic. For in asserting that $\mathrm{T}^{\prime}$ is interpreted realistically, one is asserting that $\mathrm{T}^{\prime}$ postulates the existence of, and ascribes properties to, particles of unstressed radius $\mathrm{r}$. Clearly $\mathrm{T}$ ' must attribute some kind of property to the particles if they are to be differentiated from empty space. Let us suppose that $T$ ' attributes elasticity $E$ to the particles. But the assertion that a certain particle has elasticity E (with Young's modulus $=Y$, Poisson's ratio $=\sigma$ ) must imply that that particle 'obeys' the postulates (ia) and (ib). And this in turn implies that (ia) and (ib) are analytic statements, since the meaning of 'elasticity E' presupposes the truth of (ia) and (ib). Furthermore the meaning of 'elasticity E' must presuppose the truth of some 'conservation' postulate concerning elasticity, for if the elasticity of a particle varied in an arbitrary manner, it would be meaningless to attribute elasticity $\mathrm{E}$ to that particle at some instant.

To this it might be objected that in attributing the dispositional property of elasticity to the particles, one might not mean anything so precise as that the particles 'obey' (ia) and (ib). One might mean simply that when the particles collide, they rebound, momentarily altering their shape.

But what possible justification can there be for maintaining that at any instant an object can only have a somewhat vague dispositional property? How could one decide whether a given dispositional property is sufficiently vague to be capable of existing at an instant? Clearly the thesis that 'elasticity $\mathrm{E}$ ' is too precise a dispositional property to be capable of being possessed by a particle at an instant, must be rejected.

Once it is acknowledged that a particle can have the dispositional property of elasticity at an instant, what justification can there be for denying that the particles can have the dispositional properties of mass, 'electrical' charge and gravitational charge? If a particle can 
have, at an instant, a property which can only be referred to if (i) constitutes an analytic statement, why should not the particles have, at an instant, properties which can only be referred to if (ii)-(vi) are analytic statements?

In short, once it is granted that $\mathrm{T}$ can legitimately be interpreted realistically, there can be no more reason for denying that the particles, postulated by T, can have, at any given instant, the dispositional properties of elasticity, mass, 'electrical' charge and gravitational charge, than there can be for denying that petrol can have, at any given instant, the dispositional property of inflammability, or diamond the dispositional property of solidity.

As I have already remarked, the fact that the postulates (i)-(vi) of T are analytic does not mean that T itself is analytic, for of course (vii) is a non-analytic statement. It is true that if T is to be a falsifiable theory, then non-analytic 'bridge' statements must be added to T. But these 'bridge' statements may be regarded as falling within the province of postulate (vii), since they state, when taken together, that all the various observational entities and properties known to exist are in fact certain combinations of entities and properties postulated by $\mathrm{T}$. In other words the 'bridge' statements may be regarded as a partial, but falsifiable, rendering of the statement: The world consists entirely of particles of mass $\mathrm{m}$, charge $\pm \mathrm{q}$, unstressed radius $\mathrm{r}$, elasticity E. Clearly T can be a falsifiable theory even though postulates (i)-(vi) are all analytic.

There is a sense in which $\mathrm{T}$, if it were true, would provide an ultimate explanation of phenomena, an explanation whose basic premises could themselves require no further explanation. But, it may be objected, the task of theoretical physics can never be completed in this kind of way, for at any level there must always remain further legitimate theoretical problems to be solved. Hence it would be extremely bad policy for the physicist to formulate any theory analogous to $\mathrm{T}$ in the above respect. $\mathrm{T}$, for methodological reasons, must be characterized as a non-scientific, metaphysical theory. ${ }^{14}$

But even if $\mathrm{T}$ is true, and does, therefore, provide an ultimate explanation of phenomena, this could never be known for certain. Thus even if T meets with complete empirical success, it will still be desirable for physicists to attempt to refute $\mathrm{T}$, to attempt to devise theories which are not refuted where $T$ perhaps is refuted. One could not be certain, however, that such a programme would meet with success. Just as no one can ever be certain that the 'ultimate', true physical theory has been formulated, so equally no one can be certain that the 'ultimate', true physical theory will never be formulated. I conclude that it would not be methodologically inadvisable for the physicist to formulate a theory analogous to $\mathrm{T}$, supposing such a thing were possible.

A somewhat different objection to the thesis that the truth of Timplies the truth of (1) is the following. Suppose a true, comprehensive description, D, of the universe is given in terms of $\mathrm{T}$ at some instant. Then D cannot logically imply a description of any subsequent state of affairs, for it is always logically possible, given the truth of $\mathrm{D}$, that entities should suddenly 'pop' into existence and interact with the particles described by $\mathrm{D}$.

This objection can be met if $\mathrm{T}$ is reformulated so that it, in effect, attributes dispositional properties to space, i.e. if T reformulated as a field theory. For if any point of empty space has the property that an entity can exist there only if it is a particle postulated by $\mathrm{T}$, which moves in accordance with the 'laws' of T, then at no point in space can an entity suddenly 'pop' into existence. A comprehensive description of the uni verse at some instant would not logically permit entities to 'pop' into existence at subsequent instants.

Finally it might be objected that if the argument of this section is accepted, then for any two successive events $E_{t}$ and $E_{2}$, in any universe whatsoever, (1) will be true, since it must always be possible to formulate propositions $\mathrm{P}_{1}$ which constitute a description of what exists at the time of occurrence of $E_{1}$, and which logically imply propositions $P_{2}$ which state that $E_{2}$ occurs subsequently. But if (1) is analytically true, (1) cannot be interpreted as making an existential claim.

My reply to this is as follows. If the propositions $\mathrm{P}_{1}(a)$ are to qualify as constituting no more than a description of what exists at $\mathrm{t}_{1}$, the time of occurrence of $\mathrm{E}_{1}$, and $(b)$ are to imply logically $\mathrm{P}_{2}$ then, at some level, $\mathrm{P}_{1}$ must attribute, to entities at $\mathrm{t}_{1}$, dispositional properties which cannot, by definition, change. In other words $\mathrm{P}_{1}$ must incorporate terms which (a) ascribe dispositional 
properties to entities at $\mathrm{t}_{1}(b)$ are such that it follows analytically, form the meaning of these terms, that the dispositional properties in question do not change. (Derivative dispositional properties may of course change. But, at some level, the manner and circumstances in which these dispositions change must not themselves change; and this implies the existence of higherlevel unchanging dispositional properties.) Clearly, unchanging dispositional properties cannot be truly ascribed to objects in any universe whatsoever.

IV

Finally, what are the implications of rejecting Hume's contention? This question really needs another paper. I can here do little more than indicate what seem to me to be the implications of rejecting Hume's thesis.

When one first reads Hume one is inclined to conclude, considerably shocked, that if Hume's arguments concerning the notion of 'necessary connection' are correct, then:

(7) There can be no rational grounds for the belief that the orderliness, regularity, or lawfulness that has been found in the world so far will persist in the future.

(8) Any orderliness, regularity, or lawfulness that does persist must be utterly inexplicable, must be something for which there can be no explanation whatsoever.

Hume's arguments can seem to imply this because one assumes that:

(9) There can only be rational grounds for the belief that past lawfulness will persist in the future if it is rational to believe that that which exists at one instant determines, with logical necessity, what exists subsequently.

(10) There can only be a genuine explanation of observed, persistent regularities if that which exists at one instant determines, with logical necessity, what exists subsequently.

It has often been pointed out, however, that Hume's contention does not entail (7) and (8) above. For if Hume's contention is true, then it is meaningless to assert "That which exists at one instant determines, with logical necessity, what exists subsequently". In other words the stipulations (9) and (10) become meaningless. One must therefore revise one's notions of what can constitute necessary conditions for $(a)$ rational grounds for the belief that past regularities will persist in the future, $(b)$ an explanation of persistent observed regularities.

On the other hand, if Hume's contention is false, then the stipulations (9) and (10) are no longer meaningless. Thus the need to revise one's notions of what can constitute necessary conditions for (a) and $(b)$ above collapses. Both (9) and (10) become legitimate assumptions. And it seems to me that if (9) and (10) can be accepted then they certainly ought to be accepted.

In short, once one rejects Hume's contention concerning necessary connections, then one must reject also those quasi-Humean theses whose acceptability depends on the validity of Hume's contention. It seems to me that quasi-Humean accounts of nomic universality, scientific explanation, and (a) and (b) above all fall into this category.

\section{Notes}

${ }^{1}$ D. Hume, A Treatise of Human Nature, vol. 1 (London, 1911), 163-4.

${ }^{2}$ Einstein's remark referred specifically to mathematical propositions: see A. Einstein, Geometrie und Erfahrung, Springier (Berlin, 1921), pp. 3-4.

${ }^{3}$ For a defence of what is, in effect, the thesis that the special theory of relativity is compatible with $\mathrm{C}_{2}$, see W. Sellars, 'Time and the World Order', in H. Feigl and G. Maxwell, eds., Scientific Explanation, Space and Time, Minnesota Studies in the Philosophy of Science, vol. 3 (University of Minnesota Press, Minneapolis, 1962). [Added in 2011: I have since modified my view that $\mathrm{C}_{2}$ is required for necessary connections to be possible: see my The Comprehensibility of the Universe (Oxford University Press, 1998, 
pp. 141-155). There I argue necessary connections are possible even if $\mathrm{C}_{1}$ is true.]

${ }^{4}$ This suggestion has been defended at length by W. Sellars, see ibid.

${ }^{5}$ For recent developments in 'tense logic' (which is based on the idea that a proposition can have different truth-values at different times), see A. Prior, Past Present and Future (Oxford University Press, 1967).

${ }^{6}$ That ordinary descriptive predicates are dispositional predicates, and presuppose the truth of laws or theories, has been repeatedly emphasized by Popper: see, for example, K. Popper, The Logic of Scientific Discovery (Hutchinson, London, 1959), pp. 422-6.

${ }^{7}$ For criticisms of phenomenalism, see, for example, J. J. C. Smart, Philosophy and Scientific Realism (Routledge and Kegan Paul, London, 1963), chapter 2; and W. Sellars, Science, Perception and Reality (Routledge and Kegan Paul, 1963), chapter 3.

${ }^{8}$ For this kind of quasi-Humean approach, see E. Nagel, The Structure of Science (Routledge and Kegan Paul, London, 1961), pp. 56-73.

${ }^{9}$ By 'essentialist' interpretation of a law I mean only that the law is interpreted as attributing a dispositional property — such as gravitational charge — to objects of a certain kind.

${ }^{10}$ It might be noted that if there is a perfectly straightforward (but non-Humean) sense in which only 'essentialist' laws can assert 'nomic' universality, there is also a perfectly straightforward (but non-Humean) sense in which only 'essentialist' laws can qualify as explanatory laws.

${ }^{11}$ Of course in particular cases there may well be valid objections to interpreting a physical theory realistically: many valuable physical theories, if interpreted realistically, become incompatible with other accepted physical theories, or internally inconsistent.

${ }^{12}$ One main objection to the thesis that in general physical theories cannot be interpreted realistically is that this thesis depends on the possibility of making a general, precise, non-arbitrary distinction between theoretical and observational terms, and no such distinction is, it seems, possible. One main reason why it is desirable to interpret physical theories realistically is this: there is an important sense in which only an 'essentialist' - and hence realistic - theory can be an explanatory theory (see above, $n$. 10). ${ }^{13}$ For two recent accounts of 'bridge' statements along these lines, see K. F. Schaffner, 'Approaches to Reduction', Philosophy of Science, vol. 34 (June 1967), pp. 137-47; L. Sklar, Types of Inter-Theoretic Reduction', British Journal for the Philosophy of Science, vol. 18 (1967), pp. 109-24.

${ }^{14}$ Popper, for example, has argued that ultimate, or what he calls 'essentialist', explanations ought to be excluded, for methodological reasons, from science; see K. Popper, Conjectures and Refutations (Routledge and Kegan Paul, London, 1963), pp. 104-7. 